\title{
Article \\ Polymer Microparticles Prolong Delivery of the 15-PGDH Inhibitor SW033291
}

\author{
Alan B. Dogan ${ }^{1}$ (D), Nathan A. Rohner ${ }^{1}$, Julianne N. P. Smith ${ }^{2}$, Jessica A. Kilgore ${ }^{3}$, Noelle S. Williams ${ }^{3}$, \\ Sanford D. Markowitz ${ }^{2,4}$, Horst A. von Recum ${ }^{1,+}$ and Amar B. Desai ${ }^{2, *,+}$ (D) \\ 1 Department of Biomedical Engineering, Case Western Reserve University, Cleveland, OH 44106, USA \\ Abd51@case.edu (A.B.D.); nathanrohner@gmail.com (N.A.R.); hav1@case.edu (H.A.v.R.) \\ 2 Department of Medicine and Case Comprehensive Cancer Center, Case Western Reserve University, \\ Cleveland, OH 44106, USA; Julianne.noel@gmail.com (J.N.P.S.); sxm10@case.edu (S.D.M.) \\ 3 Department of Biochemistry, UT Southwestern Medical Center, Dallas, TX 75390, USA; \\ Jessica.Kilgore@utsouthwestern.edu (J.A.K.); Noelle.Williams@utsouthwestern.edu (N.S.W.) \\ 4 University Hospitals Seidman Cancer Center, Cleveland, OH 44106, USA \\ * Correspondence: abd10@case.edu \\ + These authors contributed equally to this work.
}

check for updates

Citation: Dogan, A.B.; Rohner, N.A.; Smith, J.N.P.; Kilgore, J.A.; Williams, N.S.; Markowitz, S.D.; von Recum,

H.A.; Desai, A.B. Polymer Microparticles Prolong Delivery of the 15-PGDH Inhibitor SW033291.

Pharmaceutics 2022, 14, 85.

https://doi.org/10.3390/

pharmaceutics14010085

Academic Editor: Paola Mura

Received: 3 November 2021

Accepted: 21 December 2021

Published: 30 December 2021

Publisher's Note: MDPI stays neutral with regard to jurisdictional claims in published maps and institutional affiliations.

Copyright: (c) 2021 by the authors Licensee MDPI, Basel, Switzerland. This article is an open access article distributed under the terms and conditions of the Creative Commons Attribution (CC BY) license (https:/ / creativecommons.org/licenses/by/ $4.0 /)$.

\begin{abstract}
As the prevalence of age-related fibrotic diseases continues to increase, novel antifibrotic therapies are emerging to address clinical needs. However, many novel therapeutics for managing chronic fibrosis are small-molecule drugs that require frequent dosing to attain effective concentrations. Although bolus parenteral administrations have become standard clinical practice, an extended delivery platform would achieve steady-state concentrations over a longer time period with fewer administrations. This study lays the foundation for the development of a sustained release platform for the delivery of (+)SW033291, a potent, small-molecule inhibitor of the 15-hydroxyprostaglandin dehydrogenase (15-PGDH) enzyme, which has previously demonstrated efficacy in a murine model of pulmonary fibrosis. Herein, we leverage fine-tuned cyclodextrin microparticles-specifically, $\beta$-CD microparticles ( $\beta$-CD MPs) - to extend the delivery of the 15-PGDH inhibitor, $(+)$ SW033291, to over one week.
\end{abstract}

Keywords: fibrosis; 15-PGDH; SW033291; cyclodextrin; affinity

\section{Introduction}

In a normal wound healing response, fibroblast activity is essential for remodeling the extracellular matrix (ECM) after injury. However, in instances of unbalanced or uncontrolled tissue remodeling, the excessive deposition of ECM components results in fibrosis, which is a common pathological outcome of several chronic inflammatory diseases [1]. During fibrosis, connective tissue replaces parenchymal tissue, rendering the inflicted tissue partially or completely inflamed or damaged. Clinically, the most common manifestations of fibrotic damage can be seen in chronic diseases such as end-stage liver failure, kidney disease, heart failure, and idiopathic pulmonary fibrosis (IPF) [2].

Markedly, IPF, a chronic interstitial lung disease characterized by dyspnea, cough and increasing immobility, is particularly prevalent in 60-75-year-old patients with a history of smoking and/or occupational exposure to inhaled hazards [3]. Although the disease is expected to increase in prevalence in an increasingly aging population, current treatment options for IPF only extend the average life to $9-11$ years after diagnosis $[4,5]$. To date, two drugs have been clinically approved for IPF-pirfenidone and nintedanib. However, both small-molecule drugs suffer from short half-lives, adverse drug reactions, high costs, and frequent oral dosing (TID, $3 \times$ daily and BID, $2 \times$ daily, respectively) [6,7]. Additionally, a large percentage of elderly patients discontinue taking these drugs due to gastrointestinal and other off-target complications [8]. 
Inhibition of 15-PGDH, the primary enzyme responsible for Prostaglandin E2 metabolism, using the small molecule (+)SW033291, has recently demonstrated preclinical efficacy in a murine model of bleomycin-induced IPF by limiting systemic inflammatory load and reducing pulmonary collagen deposition $[9,10]$. Further preclinical optimization would involve ensuring that (+)SW033291 can be effectively delivered to the region of treatment with minimized off-target action. Therefore, we propose to expand the clinical utility of 15-PGDH inhibition (PGDHi) by developing a delivery system to administer sustained PGDHi in chronic disease such as IPF. We predict that sustained 15-PGDH inhibition via injectable, cyclodextrin-derived microparticles will be a novel tolerated strategy to not only reduce fibrotic deposition and decrease morbidity and mortality in murine pulmonary fibrosis, but to also reduce the demands on the patient to comply with therapeutic dosing regimens, with clinical implications for a number of additional fibrotic conditions.

To this end, we propose the use of crosslinked cyclodextrin microparticles (CD MPs) as a vehicle for the sustained release of the 15-PGDH inhibitor (+)SW033291. CD systems have previously been shown to enhance the solubility and bioavailability of drugs by complexing small-molecule, hydrophobic payloads within its interior pocket, forming an 'inclusion complex' [11]. Furthermore, CD systems with high densities of neighboring inclusion complexes are able to leverage their thermodynamic interactions with the payload to yield a controlled-release delivery system that can deliver drug for 28-70 days [12-16]. Our group has also shown that implanted cyclodextrin-based systems have the potential to 'refill' after being exposed to a second bolus dose in situ, which would present the opportunity for an adjustable and tunable window of drug delivery, as opposed to similar polymeric delivery systems which are single-use, such as PLGA [14,16].

We believe that based on its molecular structure, SW033291 could be a compatible drug for CD platforms, and that the combination device (CD MPs, loaded with SW033291) will require less drug to achieve minimally effective concentrations and will reduce off-target effects in at-risk patient populations, ultimately improving patient outcomes in IPF. This is the first study to investigate the potential pairing of SW033291 with a cyclodextrin-based delivery system, and will hopefully lay the foundations for future studies investigating the efficacy of an SW033291/cyclodextrin combination product to extend drug delivery duration and localize SW033291's physiological effects. Herein, we investigate and characterize the synthesis, loading, in vitro kinetics, and preliminary in vivo tolerance of CD MPs loaded with SW033291 in murine models to work towards a novel, sustained 15-PGDH inhibitor delivery system.

\section{Materials and Methods}

\subsection{Materials}

Soluble $\beta$-cyclodextrin ( $\beta-C D)$, lightly cross-linked with epichlorohydrin, referred to in this study as 'prepolymer', was purchased from CycloLab (Budapest, Hungary). Ethylene glycol diglycidyl ether (EGDE) crosslinker was purchased from Polysciences, Inc. (Warrington, PA, USA). (+)SW033291 was provided by Dr. Sanford Markowitz (Case Western Reserve University). The Lad2 cell line was provided by Dr. Dean Metcalfe (NIAID). Phosphate-buffered saline $(1 \times$ PBS) was purchased from Millipore Sigma (Burlington, MA, USA). Corning transwell plates and all other reagents, solvents, and chemicals were purchased from Thermo Fisher Scientific (Hampton, NH, USA) in the highest grade available.

\subsection{In Silico "Affinity" Predictions: Cyclodextrin and SW033291}

SW033291 was determined to be an appropriate fit for CD delivery platforms through in silico analysis, utilizing both molecular docking software, PyRx v. 0.9.7, running the Autodock Vina algorithm (https:/ / pyrx.sourceforge.io/, accessed on 7 October 2021, Released 2017, Molecular Graphics Laboratory, The Scripps Research Institute, La Jolla, CA, USA) and a cyclodextrin affinity predictor algorithm, developed by our group [17]. Molecular structure data files for SW033291 were downloaded from PubChem and cyclodextrin variants $(\alpha-C D, \beta-C D$, and $\gamma-C D)$ were isolated using Jmol v. 14 (http:/ / www.jmol.org/, 
accessed on 7 October 2021) from Protein Data Bank entries 1CXF, 3CGT, and 1D3C, respectively. Previous studies have shown that "binding affinity", represented by $K_{D}$, is closely correlated with the duration of drug delivery, and that drug complexation with CD does not impact the chemical properties of the drug payload [11,18-20].

\subsection{Cyclodextrin Microparticle Synthesis and Characterization}

Similar to previously established protocols for synthesizing $\beta-C D$ microparticles $(\beta-C D$ MPs), the epichlorohydrin-crosslinked $\beta$-cyclodextrin prepolymer was solubilized in $0.2 \mathrm{M}$ potassium hydroxide $(25 \% \mathrm{w} / \mathrm{v})$ and heated to $60{ }^{\circ} \mathrm{C}$ for $10 \mathrm{~min}$ [14,21-23]. Light mineral oil was warmed in a beaker with a Tween85/Span85 solution (24\%/76\%) and stirred at $500 \mathrm{rpm}$. Ethylene glycol diglycidyl ether (EDGE) was added dropwise, and the solution was vortexed for 2 min before adding the crosslinking solution to the oil/Span85/Tween85 mixture. During crosslinking $(3 \mathrm{~h})$, temperature and mixing speed were maintained at either 'Method $\mathrm{A}^{\prime}$ : $70{ }^{\circ} \mathrm{C}$ at $650 \mathrm{rpm}$ or 'Method $\mathrm{B}^{\prime}: 60{ }^{\circ} \mathrm{C}$ at $1500 \mathrm{rpm}$.

The microparticles were then centrifuged at $200 \times g$ to be separated from the oil mixture and washed with excess hexanes twice, excess acetone twice, and finally, deionized water (diH2O) twice. The microparticles were resuspended in $\mathrm{diH}_{2} \mathrm{O}$, frozen, and lyophilized for $72 \mathrm{~h}$.

Particle size was determined by a Nikon Eclipse TE300 inverted microscope (Nikon Inc., Tokyo, Japan) and analyzed for particle diameter in ImageJ. Previous studies have cited particle sizes of $81.88 \pm 36.86 \mu \mathrm{m}$ with 'Method A' synthesis [22].

Some particle groups were also crushed with a mortar and pestle for 2-3 min-referred to as 'crushed' — which is intended to reduce particle size after synthesis by $\sim 70-80 \%$ [22].

\subsection{In Vitro Reduction in Lad2-Generated 15-PGDH Due to SW033291-Loaded $\beta$-CD MPS}

The in vitro activity of SW033291 loaded in $\beta$-CD MPs was assessed by treating the Lad2 cell line and assessing the enzyme inhibition activity, in a previously described assay [24,25]. Lad 2 cells have previously been shown to highly express 15-hydroxyprostaglandin dehydrogenase (15-PGDH); therefore, they were chosen as a model cell-line for assessing PGDHi [24,26]. Briefly, either $1 \mathrm{~mL}$ of $600 \mu \mathrm{M}$ SW033291 (25\% DMSO in 2\% FBS media) or $20 \mathrm{mg} / \mathrm{mL}$ loaded $\beta$-CD MPs ( $2 \%$ FBS media) were added to the top portion of the $0.4 \mu \mathrm{m}$ pore Transwell plate. The bottoms of the plate were seeded with $200 \mathrm{k} / \mathrm{mL}$ LAD2 cells in $2 \mathrm{~mL} 2 \%$ FBS media and the plates were incubated at $37^{\circ} \mathrm{C}$ in static conditions. At each time point, $1 \mathrm{~mL}$ of media was sampled from the bottom wells, $0.1 \mathrm{~mL}$ of solution was sampled from the top well and replaced with fresh media, and the top wells were transferred to a fresh plate. Enzyme activity from bottom media samples was recorded as counts per minute (CPM) over one hour and normalized to protein input mass (mg).

For each well, $20 \mathrm{mg} / \mathrm{mL} \beta-C D$ MPs were loaded for $24 \mathrm{~h}$ in $250 \mu \mathrm{M}$ SW033291, washed twice with $\mathrm{diH}_{2} \mathrm{O}$, and resuspended in media.

\subsection{Liquid Chromatography/Mass Spectroscopy Procedure for SW033291-Loaded $\beta-C D$ MPs Analysis}

SW033291 concentrations in sampled aliquots were determined via LC-MS/MS in the Preclinical Pharmacology Core at UT Southwestern. Then, $0.1 \mathrm{~mL}$ of each sample was incubated at room temperature for $10 \mathrm{~min}$ with a twofold volume of acetonitrile, containing $0.1 \%$ formic acid and $50 \mathrm{ng} / \mathrm{mL}$ tolbutamide to induce solution crashing. Once precipitation occurred, samples were spun at $16,100 \times g$ for $5 \mathrm{~min}$ and the supernatant was transferred to a new tube and spun again. The supernatant was transferred to HPLC vials with inserts and analyzed by LC-MS/MS (Sciex 3200 QTRAP mass spectrometer coupled to a Shimadzu Prominence LC). An Agilent C18 XDB column (5 micron packing $50 \times 4.6 \mathrm{~mm}$ ) at $1.5 \mathrm{~mL} / \mathrm{min}$ with solvents: $(\mathrm{A})$ water $+0.1 \%$ formic acid and (B) $\mathrm{MeOH}+0.1 \%$ formic acid was used for chromatography prior to introduction of the sample into the mass spectrometer. Concentrations of SW033291 were determined by comparison with a standard curve made 
by spiking a blank matrix with varying concentrations of SW033291 which were processed as for samples.

\subsection{Improvement of $\beta$-CD MPs Loading Protocol with SW033291}

A traditional drug loading procedure (single solvent, $250 \mu \mathrm{M}$ drug, $24 \mathrm{~h}$ incubation) was found to only fill approximately $20-30 \%$ of available host CD molecules within the pCD MPs. To help drive the thermodynamic loading of SW033291 into $\beta$-CD MPs, $20 \mathrm{mg}$ of dried MPs was mixed with $800 \mu \mathrm{L}$ of $20 \mathrm{mg} / \mathrm{mL}$ SW033291 in DMSO for $1.5 \mathrm{~h}$, followed by the addition of $200 \mu \mathrm{L} \mathrm{diH} \mathrm{H}_{2} \mathrm{O}$ mixed for $0.5 \mathrm{~h}$. The addition of water is designed to drive the hydrophobic SW033291 into the MPs. The final 4:1 v/v organic:polar mixtures (final concentration of $16 \mathrm{mg} / \mathrm{mL}$ SW033291) were then incubated for at least $48 \mathrm{~h}$ at $4{ }^{\circ} \mathrm{C}$. Microparticles were spun down at 10,000 rpm for $3 \mathrm{~min}$ and washed twice with $800 \mu \mathrm{L}$ media/PBS (Figure 1).
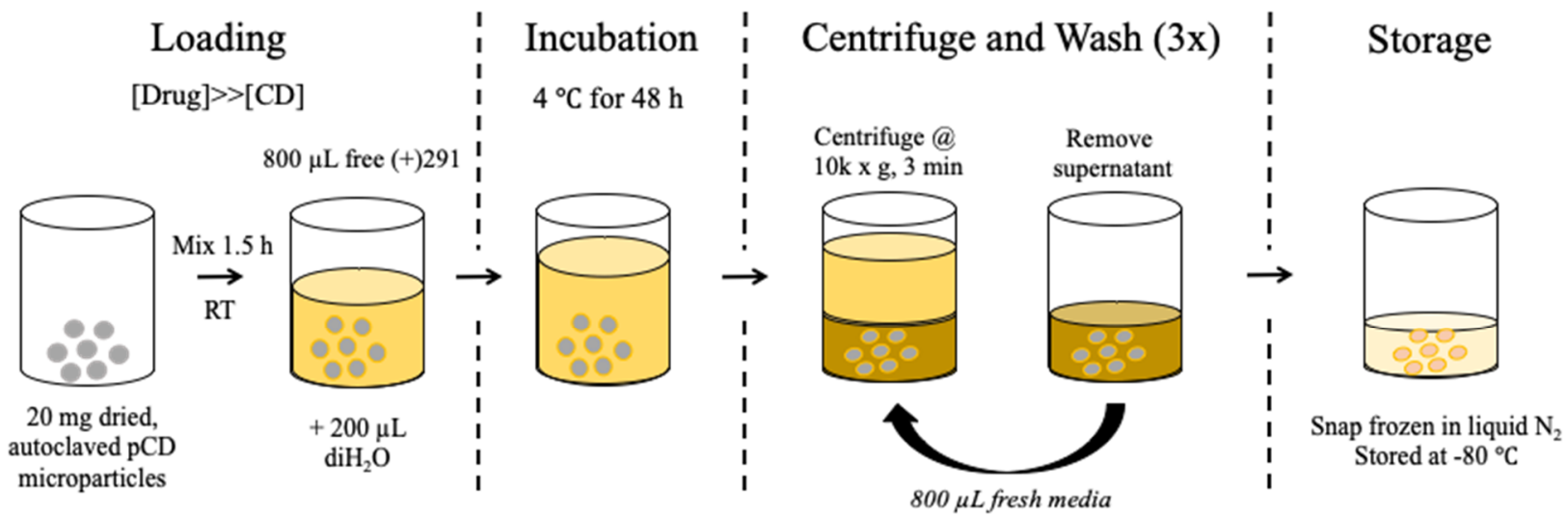

All 3 washes and the washed microparticles stored in $-80^{\circ} \mathrm{C}$. Shipped to the Preclinical Pharmacology Core for LC-MS/MS analysis at UTSW

Figure 1. Loading protocol schematic for creating SW033291-loaded $\beta$-CD MPs. Prolonging loading to $72 \mathrm{~h}$ helps ensure the complexation of SW033291 within the cyclodextrin hydrophobic 'pocket'.

\subsection{Assessing $\beta$-CD MP Injectability}

Larger diameter $\beta$-CD MPs have previously been shown to clog smaller-diameter needles. To ensure our synthesized MPs were injectable from a 29-gauge needle (BD, SafetyGlide Insulin Syringe), both the approximate settling time (seconds) and subjective injectability (binary: 'yes' or 'no') were obtained for $\beta$-CD MP formulations.

\subsection{In Vivo Tolerance Murine Model}

A murine model was used to test $\beta$-CD MP tolerance and quantify the extended delivery of SW033291 in vivo. Eight-week-old female C57/Bl6 mice were purchased from the Jackson lab (CWRU). SW033291-loaded $\beta$-CD MPs (2 mg, in $200 \mu \mathrm{L}$ PBS) were administered retro-orbitally $(\mathrm{RO})$ and peripheral blood was collected into Microtainer serum-separator tubes (Becton-Dickinson) by submandibular cheek puncture at designated time points ( $n=4$ mice per arm; 24, 72, 168, and $336 \mathrm{~h}$ post-administration). Whole blood was allowed to clot at room temperature and then spun at $6000 \times g$ for $3 \mathrm{~min}$ to separate the serum. Serum was removed and stored at $-80^{\circ} \mathrm{C}$ prior to sending samples to the Preclinical Pharmacology Core at UTSW for LC-MS/MS analysis. Analysis was conducted as described above, except the matrix utilized was mouse serum rather than culture media.

\subsection{Statistical Analysis}

Experiments were all carried out in triplicates, unless otherwise stated, and data are presented as the mean \pm standard error. In vivo data represent the average of $n=4$ mice, and error bars also represent the standard error of the mean. 


\section{Results}

\subsection{SW033291 Is Predicted to Have Significant Affinity for $\beta$-CD Host In Silico}

Before beginning in vitro studies, we confirmed that a cyclodextrin delivery platform would be compatible with (+)SW033291's chemical structure in silico. Utilizing two methods of affinity prediction-docking simulations and quantitative structure activity relationship (QSAR) — we found that SW033291 had the highest binding affinity with the $\beta$ form of cyclodextrin (Table 1). Docking simulations also revealed that the central moiety which binds with cyclodextrin includes SW033291's central heterocyclic rings and sulfoxide group (Figure 2). Based on previous studies, we predicted that a binding affinity of $-23 \mathrm{KJ} \cdot \mathrm{mol}^{-1}$ would result in a window of release of about $1-2$ weeks $[21,27-29]$.

Table 1. Affinity binding simulations of 15-PGDH inhibitor SW033291 (CID:337839) complexation with $\alpha, \beta$, and $\gamma$ cyclodextrin (CD) in both PyRx and a machine learning algorithm for affinity prediction [17].

\begin{tabular}{cccc}
\hline Ligand & Host & Binding Affinity-PyRx (KJ/mol) & Binding Affinity-ML Model (KJ/mol) \\
\hline 15-PGDH inhibitor SW033291 & $\alpha-C D$ & $-17.9 \pm 1.7$ & -15.7 \\
15-PGDH inhibitor SW033291 & $\beta-C D$ & $-23.0 \pm 1.6$ & -18.2 \\
15-PGDH inhibitor SW033291 & $\gamma$-CD & $-22.0 \pm 1.0$ & -14.3 \\
\hline
\end{tabular}
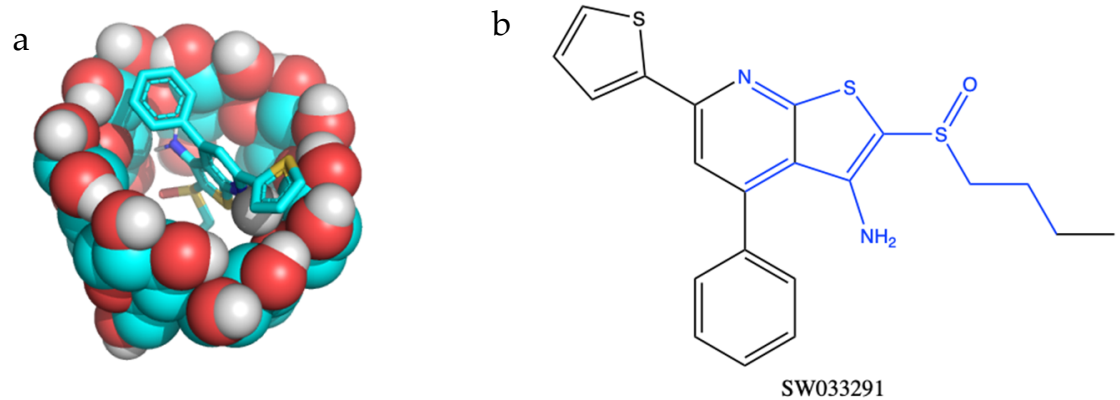

Figure 2. (a) Molecular structure in silico model demonstrating inclusion complex formation between the small-molecule drug SW033291 binding to the inner pocket of $\beta$-cyclodextrin. (b) Regions of SW033291 in blue are complexed within the cyclodextrin 'pocket'.

\subsection{Lowering the Temperature of Crosslinking and Increasing the Speed of Mixing Generates Smaller $\beta$-CD MPS}

Established protocols for synthesizing $\beta$-CD MPs generate diameters that are not suitable for small-gauge needle injections; therefore, we sought to generate MPs with smaller diameters to allow injectability (Figure 3, Table 2). To this end, we modulated parameters during polymerization-specifically, temperature and mixing speed during polymerization - to generate smaller microparticles. We also explored manually crushing MPs with a mortar and pestle, which was previously reported to reduce particle diameters by $30 \%$ [22]. By reducing the temperature and raising the mixing speed to $1500 \mathrm{rpm}$, we generated MPs $\sim 40 \mu \mathrm{m}$ in diameter, a 50\% reduction from the original protocol (Figure 4). Microparticles were then loaded for $24 \mathrm{~h}$ with (+)SW033291, and final loading concentrations were reported after drug leaching. 


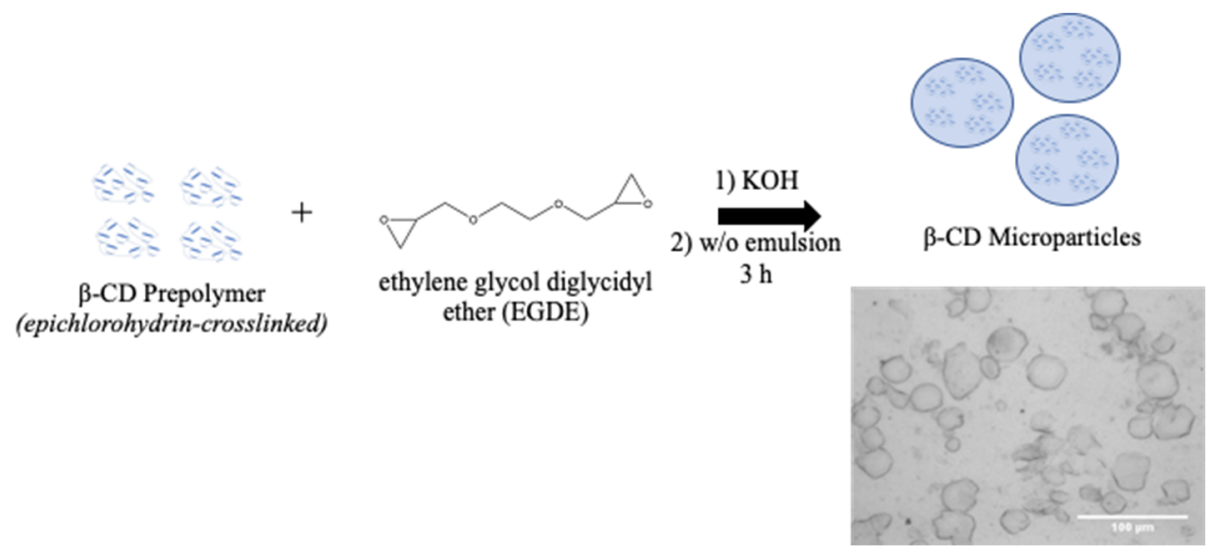

Figure 3. Overview of $\beta$-CD microparticle preparation. $\beta-C D$ prepolymer is crosslinked with EDGE for $3 \mathrm{~h}$ at elevated temperature $\left(60-70{ }^{\circ} \mathrm{C}\right)$ and formed in a water/oil $(\mathrm{w} / \mathrm{o})$ emulsion.

Table 2. Microparticle batches synthesized with varying temperature and mixing speeds. Particles were then loaded with (+)SW033291 for 24 h (DMSO). Particle diameters were determined by ImageJ, and are presented as the mean \pm S.D. Final loading concentrations were determined via LC/MS at UTSW.

\begin{tabular}{ccc}
\hline Batch Preparation & Particle Diameter $(\boldsymbol{\mu m})$ & $\begin{array}{c}\text { Final Loading Concentration } \\
(\mathbf{m g} / \mathbf{m L})\end{array}$ \\
\hline Method A, crushed & $61.0 \pm 26$ & 32.9 \\
\hline Method B, uncrushed & $41.2 \pm 13$ & 12.2 \\
\hline Method B, crushed & $25.0 \pm 9.5$ & 18.0 \\
\hline Method B, crushed & $35.0 \pm 9.5$ & 19.0 \\
\hline
\end{tabular}

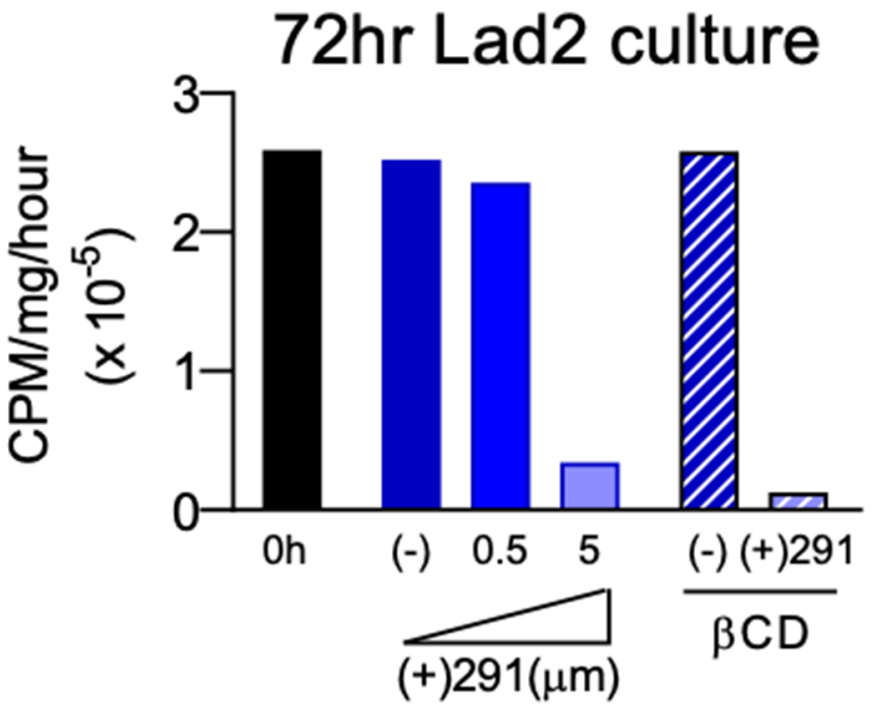

Figure 4. Incubation study of (+)SW033291-loaded $\beta$-CD MPs with Lad2 cells indicated sustained delivery in co-culture inhibits enzyme activity $72 \mathrm{~h}$ post-administration with similar efficacy as bolus SW033291 administration. (-) indicates the negative control group.

\subsection{SW033291 Released from $\beta$-CD MPs Has Prolonged Lad2 Inactivation Compared to Free Drug}

To confirm drug activity after delivery from $\beta$-CD MPs, (+)SW033291 activity was analyzed in vitro by proxy of analyzing supra-physiological concentrations of Lad2 enzyme activity inhibition. Using Method A, with uncrushed particles (final loading concentration: 
$11.9 \mathrm{mg} / \mathrm{mL}$ ), we found that $\beta-C D$ MP-released (+)SW033291 was effective at reducing LAD2 enzyme activity comparable to $5 \mu \mathrm{M}$ free drug after $72 \mathrm{~h}$ (Figure 4 ). LAD2 controls and LAD2 treated with unloaded $\beta$-CD MP controls saw unremarkable changes in enzyme activity, confirming that $\beta-C D$ alone does not contribute to enzyme inactivation. Drug release kinetics were also observed to exhibit increasingly zero-order kinetics compared to bolus administration, confirming that $\beta$-CD MPs slowed the rate of release of (+)SW033291 over a three-day period (Figure S2).

\section{4. $\beta$-CD MP Loading Optimization}

To help address the relatively low observed 'final loading concentration' seen in the $24 \mathrm{~h}$ loaded $\beta-\mathrm{CD} \mathrm{MP}$, we altered the loading protocol to encourage increased packing of drug within the microparticles. Comparing ' $24 \mathrm{~h}$ loading', which used $24 \mathrm{~h}$ incubation in (+)SW033291 dissolved in DMSO, with '72 h loading', which has previously been described as mixing a polar and nonpolar solvent to encourage complexation of the hydrophobic drug within the CD pockets. We found that altering this procedure yielded final loading concentrations 4- to 14-fold greater compared with our previous protocol (Figure 5).

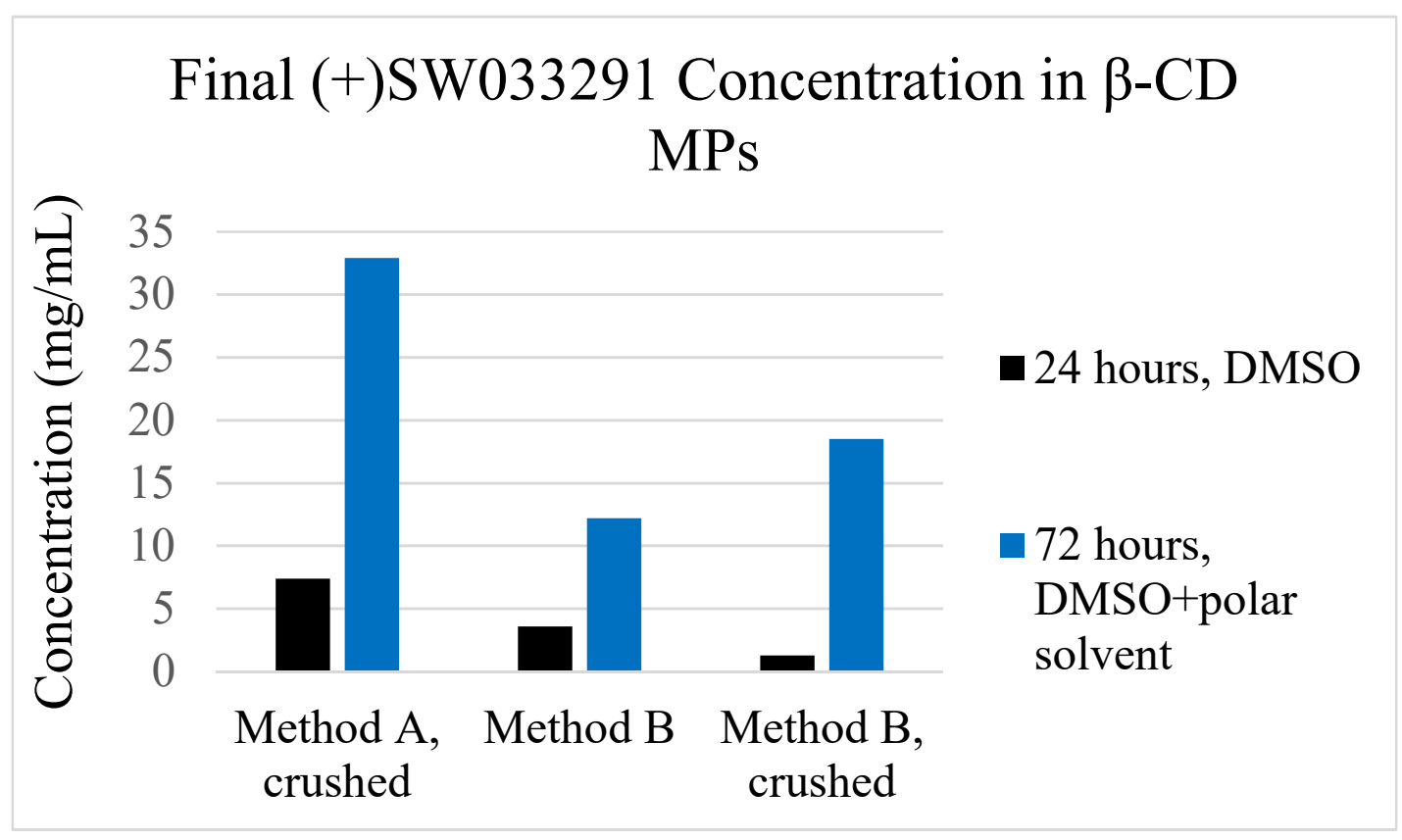

Figure 5. Final loading concentrations for $\beta$-CD MP formulations utilizing a ' $24-\mathrm{h}$ ' loading protocol and our investigated '72-h' loading protocol, which uses loading solution of both DMSO and a polar solvent (water) to help drive complexation (referred to as "DMSO + polar solvent").

\subsection{At Lower Concentrations, $\beta$-CD MPs Are Injectable but Are Prone to Clumping}

Before moving to an in vivo model, we aimed to investigate whether smaller-diameter $\beta-C D$ MPs are suitable for injection from 29-Gauge syringes. In a serial dilution of $40 \mathrm{mg} / \mathrm{mL}$, which was determined to be the maximum concentration of MPs achievable in an aqueous medium without immediate crashing, we found that the formulations were injectable $\leq 10 \mathrm{mg} / \mathrm{mL}$; however, issues with clumping were observed (Table 3). At a lower concentration of $\leq 1.3 \mathrm{mg} / \mathrm{mL}$, there was notably less clotting observed. Moving forward in vivo, we selected $10 \mathrm{mg} / \mathrm{mL}$ in order to ensure maximum $\beta$-CD MP delivery in a suitable volume $(\sim 200 \mu \mathrm{L})$. 
Table 3. Serial dilution of $\beta-C D$ MPs in 1x PBS. Approximate settling times were measured subjectively when depositions of microparticles were first observed to crash out from solution. 'Injectability' was rated as a binary 'yes' or 'no' qualitative observation.

\begin{tabular}{ccc}
\hline $\begin{array}{c}\text { Concentration of } \beta-C D ~ M P s \\
(\mathbf{m g} / \mathbf{m L} \mathbf{d}=\mathbf{3 5} \boldsymbol{\mu m})\end{array}$ & $\begin{array}{c}\text { Approximate Settling Time } \\
\mathbf{( s )}\end{array}$ & $\begin{array}{c}\text { Injectable? } \\
(\mathbf{2 9} \mathbf{G} \text { Needle) }\end{array}$ \\
\hline 40 & 5 & no \\
20 & 9 & no \\
10 & 16.6 & yes \\
5.2 & 20 & yes \\
2.6 & 23 & yes \\
1.3 & 30 & yes \\
0.7 & $>60$ & yes \\
\hline
\end{tabular}

3.6. (+)SW033291-Loaded $\beta$-CD MPs Prolonged Payload Delivery over 1 Week and Were Well Tolerated in a Murine Model

SW033291-loaded $\beta$-CD MPs ( $2 \mathrm{mg}$, in $200 \mu \mathrm{L}$ PBS) were administered retro-orbitally (RO) to 8-week-old female C57/Bl6 mice $(80 \mathrm{mg} / \mathrm{kg})$ and peripheral blood was collected into Microtainer serum-separator tubes (Becton-Dickinson) by submandibular cheek puncture at designated time points $(n=4$ mice per arm; $24,72,168$, and $336 \mathrm{~h}$ postadministration). Within the cohort of 16 mice, we observed no adverse events following $\mathrm{RO}$ administration within the 2-week study. Serum drug concentrations $(n=4$ at each timepoint) revealed that (+)SW033291 was still present after 1 week post-administration of SW033291 loaded $\beta$-CD MPs, eventually becoming undetectable in three out of four mice at the 2-week timepoint (Figure 6).

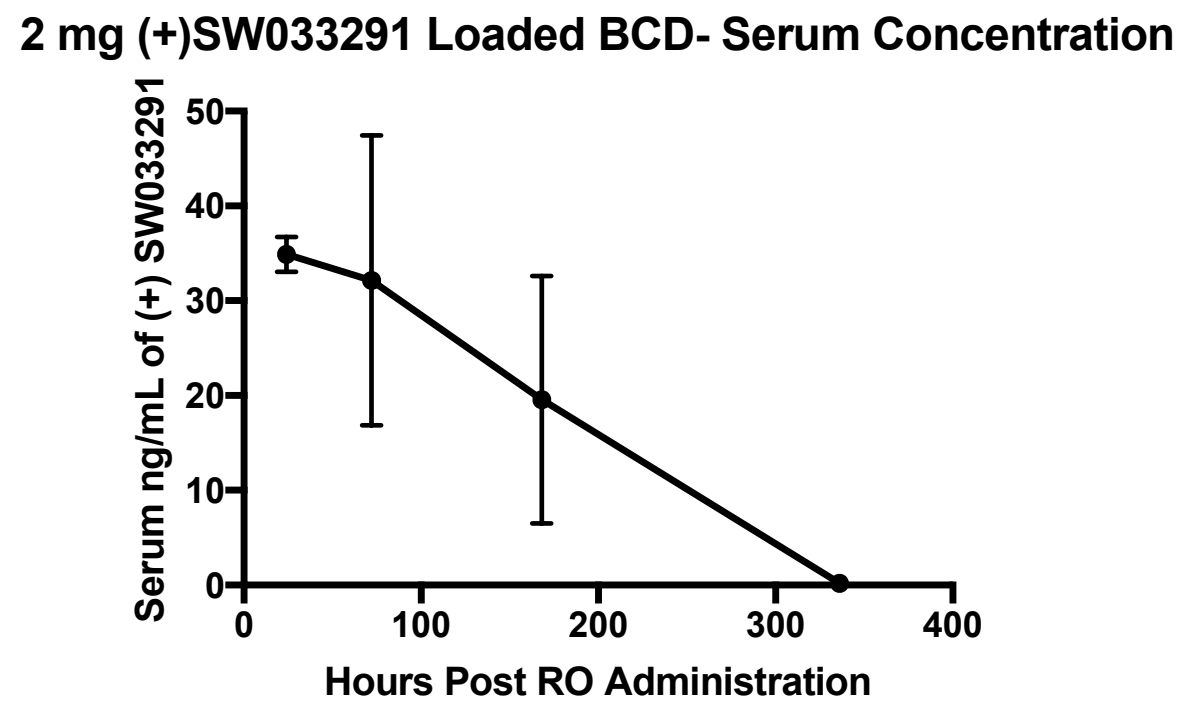

Figure 6. Serum (+)SW033291 concentrations collected from mice ( $n=4$ at each timepoint) following RO administration of (+)SW033291 loaded $\beta$-CD MPs. Error bars are representative of the standard error of the mean.

\section{Discussions}

Although pCD delivery systems have been used to extend the delivery of other smallmolecule therapeutics, this is the first study showing that $\mathrm{pCD}$ is a compatible sustained release platform for PGDHi by (+)SW033291. Using in silico methods for affinity prediction, we found that SW033291's structure was most compatible with $\beta$-CD (Figure 2). We found that $(+)$ SW033291- $\beta-C D$ interactions had a comparable free energy of binding to existing drugs that have been successfully delivered from $\mathrm{pCD}$ platforms (e.g., doxorubicin) (Figure S1). We then fine-tuned existing synthesis protocols for our lab's $\beta$-CD MPs, generating a smaller-diameter microparticle of around 30-35 $\mu \mathrm{m}$ (Figure 3), confirming 
that decreasing the temperature and increasing the rate of stirring during crosslinking in a water/oil emulsion generates smaller $\mathrm{PCD}$ particles, helping to build on previous work $[21,22]$.

Effective delivery of (+)SW033291 from $\beta$-CD MPs was also confirmed, first in vivo by effectively reducing the supra-physiological enzyme activity in LAD2 cells (Figure 4), and secondly in vivo in healthy mice (Figure 6). Drug kinetics in vitro revealed that (+)SW033291 was taking advantage of pCD inclusion, because the time of release was extended up to 6 days-compared with a 1-day bolus input-and exhibited increasingly zero-order kinetics (Figure S2).

(+)SW033291 loading protocols for $\beta$-CD MPs were also improved after discovering that $24 \mathrm{~h}$ incubation times in DMSO were insufficient to promote drug inclusion. To this end, we modified existing loading protocols by extending incubation time to $72 \mathrm{~h}$ and adding an aqueous component to the loading solution to help promote drug inclusion to $\mathrm{pCD}$ pockets (Figures 1 and 5). This further confirms that drug inclusion within cyclodextrin and other similar drug delivery systems is highly dependent on solvent contact time and respective solubility of the drug within the drug-loading solvent [30-32].

It has previously been reported that cyclodextrin-derived polymers are relatively well tolerated in vivo; however, we found that $2 \mathrm{mg}$ of $\beta$-CD MPs administered retro-orbitally was tolerated without ramifications in all mice within our cohort (Figure 6) [33]. We also observed that a single dose of (+)SW033291-loaded $\beta$-CD MPs was able to produce a drug delivery profile of over 1 week in vivo (Figure 6).

This study represents the groundwork for developing an extended drug delivery system for anti-fibrotic therapies. Although we were able to access global blood serum levels of (+)SW033291, future studies will focus on analyzing the biodistribution of the $\beta$ CD MPs and whether or not drug localization is achieved in regions of interest, specifically in the pulmonary tract and hepatic system [25]. The window of delivery that was achieved in vivo greatly surpassed bolus injection; however, the amount of drug released from $2 \mathrm{mg}$ most likely would be subtherapeutic. Therefore, we propose to increase the frequency of $\beta$-CD MP administration in future models to help ensure that drug concentrations surpass minimally effective concentrations. We also aim to reduce MP clumping during administration, in which either administration protocols or synthesis quality (reducing polydispersity) can be changed to make MP injections easier [34]. In addition, altering the physical properties of the injection solution may allow for particles to remain in suspension for a longer time and might be better tolerated in vivo [35]. Ultimately, we aim to further optimize the pCD platform for the sustained delivery of (+)SW033291 and assess the efficacy of sustained PGDHi delivery as a therapeutic strategy in multiple models of chronic fibrotic disease.

\section{Conclusions}

Herein, we present the foundation for creating an injectable, cyclodextrin-based microparticle system for sustained 15-PGDH inhibition. As age-related fibrosis increases in prevalence, the long-term delivery of antifibrotic therapeutics will greatly improve upon current standards of care and augment the clinical usability of potent small-molecule fibrosis inhibitors, such as (+)SW033291. Extending the delivery of (+)SW033291 for over one week in vivo represents the first step in creating a novel tolerated strategy to reduce fibrotic deposition and improve morbidity and mortality in murine pulmonary and hepatic fibrosis. Future success in prolonged (+)SW033291 delivery would also have additional clinical implications for a number of fibrotic conditions.

Supplementary Materials: The following are available online at https: / www.mdpi.com/article/ 10.3390/pharmaceutics14010085/s1, Figure S1: Affinity prediction machine learning model with training set (black) and highlighted drugs ("molecules of interest") that were previously compatible with $\beta$-CD platforms. The dotted orange line is representative of SW033291's predicted affinity to $\beta$-CD based on chemical descriptors. All chemical descriptor information for both host and ligand was sourced from PubChem, Figure S2: Drug release kinetics $(n=1)$ sampled from top transwell 
plates for both bolus-administered SW033291 and $\beta$-CD MPs loaded with SW033291 (incubated for $24 \mathrm{~h})$. Trend lines represent a best linear fit, and trends with $\mathrm{R}^{2}$ values closer to 1 were considered "zero-order".

Author Contributions: H.A.v.R. and A.B.D. (Amar B. Desai) devised the project. A.B.D. (Alan B. Dogan) developed the technical procedures and performed the in vitro experiments under the supervision of H.A.v.R. and A.B.D. (Amar B. Desai); in vitro work and technical procedures were performed by N.A.R. and J.N.P.S.; in vivo work was completed by A.B.D. (Amar B. Desai); J.A.K. evaluated the concentrations of (+)SW033291 for both in vitro and in vivo analyses under the supervision of N.S.W.; S.D.M. assisted in experimental design and technical troubleshooting; under the supervision of H.A.v.R. and A.B.D. (Amar B. Desai), A.B.D. (Alan B. Dogan) wrote the manuscript; all authors read or edited the manuscript. All authors have read and agreed to the published version of the manuscript.

Funding: This work was supported by NIH grants 5R00HL135740-04, R35 CA197442, and by the Radiation Resources Core Facility (P30CA043703) of Case Western Reserve University, as well as the Preclinical Pharmacology Core of UT Southwestern.

Institutional Review Board Statement: Mice studies were conducted in the Case Animal Resource Center with animal care and procedures performed under protocol 2019-0065 approved by and in accordance with guidelines of Case Western Reserve University's Institutional Animal Care and Use Committee.

Informed Consent Statement: Not applicable.

Data Availability Statement: The data presented in this study are available within this article. Any additional requests for data will be promptly responded to by the corresponding author.

Acknowledgments: We thank Joseph Ready of UT Southwestern for performing QC on (+)SW033291 and providing additional scientific insight.

Conflicts of Interest: H.A.v.R. is a co-founder of Affinity Therapeutics but does not receive a salary. Amar B.D. and S.D.M. hold patents relating to the use of 15-PGDH inhibitors that have been licensed to Rodeo Therapeutics (acquired by Amgen). S.D.M. is a founder of Rodeo Therapeutics, and S.D.M. and Amar B.D. are consultants to Amgen. Conflicts of interest are managed according to institutional guidelines and overseen by Case Western Reserve University. No conflict of interest pertain to any of the remaining authors.

\section{References}

1. Murtha, L.A.; Schuliga, M.J.; Mabotuwana, N.S.; Hardy, S.A.; Waters, D.W.; Burgess, J.K.; Knight, D.A.; Boyle, A.J. The Processes and Mechanisms of Cardiac and Pulmonary Fibrosis. Front. Physiol. 2017, 8, 777. [CrossRef]

2. Wynn, T.A.; Ramalingam, T.R. Mechanisms of fibrosis: Therapeutic translation for fibrotic disease. Nat. Med. 2012, 18, 1028-1040. [CrossRef]

3. Leuschner, G.; Klotsche, J.; Kreuter, M.; Prasse, A.; Wirtz, H.; Pittrow, D.; Frankenberger, M.; Behr, J.; Kneidinger, N.; Group, T.I.I.R. Idiopathic Pulmonary Fibrosis in Elderly Patients: Analysis of the INSIGHTS-IPF Observational Study. Front. Med. 2020, 7, 601279. [CrossRef]

4. Maher, T.M.; Strek, M.E. Antifibrotic therapy for idiopathic pulmonary fibrosis: Time to treat. Respir. Res. 2019, 20, 205. [CrossRef] [PubMed]

5. Somogyi, V.; Chaudhuri, N.; Torrisi, S.E.; Kahn, N.; Müller, V.; Kreuter, M. The therapy of idiopathic pulmonary fibrosis: What is next? Eur. Respir. Rev. 2019, 28, 190021. [CrossRef] [PubMed]

6. Hajari Case, A.; Johnson, P. Clinical use of nintedanib in patients with idiopathic pulmonary fibrosis. BMJ Open Respir. Res. 2017, 4, e000192. [CrossRef]

7. Kim, E.S.; Keating, G.M. Pirfenidone: A Review of Its Use in Idiopathic Pulmonary Fibrosis. Drugs 2015, 75, 219-230. [CrossRef]

8. Naqvi, M.; D'Ancona, G.; West, A. A Comparison of Pirfenidone Versus Nintedanib for the Management of Idiopathic Pulmonary Fibrosis. In Idiopathic Interstitial Pneumonias; PA4791; European Respiratory Society: Lausanne, Switzerland, 2018. [CrossRef]

9. Desai, A.; Zhang, Y.; Park, Y.; Dawson, D.M.; Larusch, G.A.; Kasturi, L.; Wald, D.; Ready, J.M.; Gerson, S.L.; Markowitz, S.D. A second-generation 15-PGDH inhibitor promotes bone marrow transplant recovery independently of age, transplant dose and granulocyte colony-stimulating factor support. Haematologica 2018, 103, 1054-1064. [CrossRef]

10. Smith, J.N.P.; Witkin, M.D.; Jogasuria, A.P.; Christo, K.F.; Raffay, T.M.; Markowitz, S.D.; Desai, A.B. Therapeutic targeting of 15-PGDH in murine pulmonary fibrosis. Sci. Rep. 2020, 10, 11657. [CrossRef] [PubMed]

11. Haimhoffer, Á.; Rusznyák, Á.; Réti-Nagy, K.; Vasvári, G.; Váradi, J.; Vecsernyés, M.; Bácskay, I.; Fehér, P.; Ujhelyi, Z.; Fenyvesi, F. Cyclodextrins in drug delivery systems and their effects on biological barriers. Sci. Pharm. 2019, 87, 33. [CrossRef] 
12. Cyphert, E.L.; Fu, A.S.; von Recum, H.A. Featured Article: Chemotherapeutic delivery using pH-responsive, affinity-based release. Exp. Biol. Med. 2017, 242, 692-699. [CrossRef]

13. Rohner, N.A.; Nguyen, D.; von Recum, H.A. Affinity Effects on the Release of Non-Conventional Antifibrotics from Polymer Depots. Pharmaceutics 2020, 12, 275. [CrossRef] [PubMed]

14. Rohner, N.A.; Dogan, A.B.; Robida, O.A.; Von Recum, H.A. Serum biomolecules unable to compete with drug refilling into cyclodextrin polymers regardless of the form. J. Mater. Chem. B 2019, 7, 5320-5327. [CrossRef]

15. Dogan, A.; von Recum, H. Engineering selective molecular tethers to enhance suboptimal drug properties. Acta Biomater. 2020, 115, 383-392. [CrossRef]

16. Cyphert, E.L.; Learn, G.D.; Hurley, S.K.; Lu, C.; von Recum, H.A. An Additive to PMMA Bone Cement Enables Postimplantation Drug Refilling, Broadens Range of Compatible Antibiotics, and Prolongs Antimicrobial Therapy. Adv. Healthc. Mater. 2018, 7, 1800812. [CrossRef] [PubMed]

17. Kerner, J.; von Recum, H. Predicting Drug Interactions to Unassociated Biomedical Implants Using Machine Learning Techniques and Model Polymers. bioRxiv 2020. [CrossRef]

18. Kost, B.; Brzezinski, M.; Socka, M.; Basko, M.; Biela, T. Biocompatible polymers combined with cyclodextrins: Fascinating materials for drug delivery applications. Molecules 2020, 25, 3404. [CrossRef] [PubMed]

19. Creteanu, A.; Pamfil, D.; Vasile, C.; Tantaru, G.; Ghiciuc, C.M.; Ochiuz, L.; Ghilan, A.; Macsim, A.M. Study on the Role of the Inclusion Complexes with 2-Hydroxypropyl- $\beta$-cyclodextrin for Oral Administration of Amiodarone. Int. J. Polym. Sci. 2019, 2019, 1695189. [CrossRef]

20. Gao, F.; Ye, Y.J.; Wang, Y.; Lou, K.Y.; Chen, Y.Z.; Chen, R. The preparation, characterization, and pharmacokinetic studies of chitosan nanoparticles loaded with paclitaxel/dimethyl- $\beta$-cyclodextrin inclusion complexes. Int. J. Nanomed. 2015, 10, 4309. [CrossRef]

21. Rohner, N.A.; Schomisch, S.J.; Marks, J.M.; von Recum, H.A. Cyclodextrin Polymer Preserves Sirolimus Activity and Local Persistence for Antifibrotic Delivery over the Time Course of Wound Healing. Mol. Pharm. 2019, 16, 1766-1774. [CrossRef]

22. Rohner, N.A.; Purdue, L.N.; von Recum, H.A. Affinity-Based Polymers Provide Long-Term Immunotherapeutic Drug Delivery Across Particle Size Ranges Optimal for Macrophage Targeting. J. Pharm. Sci. 2021, 110, 1693-1700. [CrossRef] [PubMed]

23. Grafmiller, K.T.; Zuckerman, S.T.; Petro, C.; Liu, L.; von Recum, H.A.; Rosen, M.J.; Korley, J.N. Antibiotic-releasing microspheres prevent mesh infection in vivo. J. Surg. Res. 2016, 206, 41-47. [CrossRef] [PubMed]

24. Sekar, Y.; Befus, A.D. Protein tyrosine nitration of 15-hydroxy prostaglandin dehydrogenase in the human mast cell line LAD2. Nitric Oxide 2012, 26, 74-80. [CrossRef]

25. Zhang, Y.; Desai, A.; Yang, S.Y.; Bae, K.B.; Antczak, M.I.; Fink, S.P.; Tiwari, S.; Willis, J.E.; Williams, N.S.; Dawson, D.M.; et al. Inhibition of the prostaglandin-degrading enzyme 15-PGDH potentiates tissue regeneration. Science 2015, 348, aaa2340. [CrossRef]

26. Backlund, M.G.; Mann, J.R.; Holla, V.R.; Buchanan, F.G.; Tai, H.H.; Musiek, E.S.; Milne, G.L.; Katkuri, S.; DuBois, R.N. 15Hydroxyprostaglandin Dehydrogenase Is Down-regulated in Colorectal Cancer. J. Biol. Chem. 2005, 280, 3217-3223. [CrossRef]

27. Fu, A.S.; Thatiparti, T.R.; Saidel, G.M.; von Recum, H.A. Experimental Studies and Modeling of Drug Release from a Tunable Affinity-Based Drug Delivery Platform. Ann. Biomed. Eng. 2011, 39, 2466-2475. [CrossRef]

28. Haley, R.M.; von Recum, H.A. Localized and targeted delivery of NSAIDs for treatment of inflammation: A review. Exp. Biol. Med. 2019, 244, 433-444. [CrossRef]

29. Rivera-Delgado, E.; Sadeghi, Z.; Wang, N.X.; Kenyon, J.; Satyanarayan, S.; Kavran, M.; Flask, C.; Hijaz, A.Z.; von Recum, H.A Local release from affinity-based polymers increases urethral concentration of the stem cell chemokine CCL7 in rats. Biomed. Mater. 2016, 11, 025022. [CrossRef]

30. Šoltys, M.; Kovačík, P.; Dammer, O.; Beránek, J.; Štěpánek, F. Effect of solvent selection on drug loading and amorphisation in mesoporous silica particles. Int. J. Pharm. 2019, 555, 19-27. [CrossRef]

31. Carr, A.C.; Piunova, V.A.; Maarof, H.; Rice, J.E.; Swope, W.C. Influence of Solvent on the Drug-Loading Process of Amphiphilic Nanogel Star Polymers. J. Phys. Chem. B 2018, 122, 5356-5367. [CrossRef] [PubMed]

32. Lagreca, E.; Onesto, V.; Di Natale, C.; La Manna, S.; Netti, P.A.; Vecchione, R. Recent advances in the formulation of PLGA microparticles for controlled drug delivery. Prog. Biomater. 2020, 9, 153-174. [CrossRef] [PubMed]

33. Carneiro, S.; Costa Duarte, F.I.; Heimfarth, L.; Siqueira Quintans, J.D.S.; Quintans-Júnior, L.J.; Veiga Júnior, V.F.D.; Neves de Lima, Á.A. Cyclodextrin-Drug Inclusion Complexes: In Vivo and In Vitro Approaches. Int. J. Mol. Sci. 2019, 20, 642. [CrossRef] [PubMed]

34. Busatto, C.; Pesoa, J.; Helbling, I.; Luna, J.; Estenoz, D. Effect of particle size, polydispersity and polymer degradation on progesterone release from PLGA microparticles: Experimental and mathematical modeling. Int. J. Pharm. 2018, 536, 360-369. [CrossRef] [PubMed]

35. Schwarzenbach, F.; Filipe-Santos, O.; Wang, T.; Rojas, H.E.; Granger, C.; Schwarzenbach, F. Evaluation of the impact of viscosity, injection volume, and injection flow rate on subcutaneous injection tolerance. Med. Devices Evid. Res. 2015, 8, 473. [CrossRef] [PubMed] 